\title{
Universality of Quantum Gravity Corrections
}

\author{
Saurya Das* \\ Department of Physics, University of Lethbridge, \\ 4401 University Drive, Lethbridge, Alberta - T1K 3M4, Canada \\ Elias C. Vagenas ${ }^{\dagger}$ \\ Research Center for Astronomy \& Applied Mathematics, \\ Academy of Athens, \\ Soranou Efessiou 4, GR-11527, Athens, Greece
}

\begin{abstract}
We show that the existence of a minimum measurable length and the related Generalized Uncertainty Principle (GUP), predicted by theories of Quantum Gravity, influence all quantum Hamiltonians. Thus, they predict quantum gravity corrections to various quantum phenomena. We compute such corrections to the Lamb Shift, the Landau levels and the tunnelling current in a Scanning Tunnelling Microscope (STM). We show that these corrections can be interpreted in two ways: (a) either that they are exceedingly small, beyond the reach of current experiments, or (b) that they predict upper bounds on the quantum gravity parameter in the GUP, compatible with experiments at the electroweak scale. Thus, more accurate measurements in the future should either be able to test these predictions, or further tighten the above bounds and predict an intermediate length scale, between the electroweak and the Planck scale.
\end{abstract}

We know that gravity is universal. Anything which has energy creates gravity and is affected by it, although the smallness of Newton's constant $G$ often means that the associated effects are too weak to be measurable. In this article, we show that certain effects of Quantum Gravity are also universal, and can influence almost any system with a well-defined Hamiltonian. The resultant effects are generically quite small, being proportional to the square of the Planck length $\ell_{P l}^{2}=G \hbar / c^{3}$. However, with current and future experiments, bounds may be set on certain parameters relevant to quantum gravity, and improved accuracies could even make them measurable.

An important prediction of various theories of quantum gravity (such as String Theory) and black hole physics is the existence of a minimum measurable length. The prediction is largely model independent, and can be understood as follows: the Heisenberg Uncertainty Principle (HUP), $\Delta x \sim \hbar / \Delta p$, breaks down for energies close to the Planck scale, when the corresponding Schwarzschild radius is comparable to the Compton wavelength (both being approximately equal to the Planck length). Higher energies result in a further increase of the Schwarzschild radius, resulting in $\Delta x \approx$ $\ell_{P l}^{2} \Delta p / \hbar$. The above observation, along with a combination of thought experiments and rigorous derivations suggest that the Generalized Uncertainty Principle (GUP) holds at all scales, and is represented by [1]

$$
\begin{aligned}
\Delta x_{i} \Delta p_{i} & \geq \frac{\hbar}{2}\left[1+\beta\left((\Delta p)^{2}+<p>^{2}\right)\right. \\
& \left.+2 \beta\left(\Delta p_{i}^{2}+<p_{i}>^{2}\right)\right], i=1,2,3
\end{aligned}
$$

where $p^{2}=\sum_{j=1}^{3} p_{j} p_{j}, \beta=\beta_{0} /\left(M_{P l} c\right)^{2}=\ell_{P l}^{2} / 2 \hbar^{2}$, $M_{P l}=$ Planck mass, and $M_{P l} c^{2}=$ Planck energy $\approx$ $1.2 \times 10^{19} \mathrm{GeV}$. Implications of the GUP in various fields, including High Energy Physics, Cosmology and Black Holes, have been studied. Here, we examine its potential experimental signatures in some familiar quantum systems. It is normally assumed that the dimensionless parameter $\beta_{0}$ is of the order of unity. However, as we shall see in this article, this choice renders Quantum Gravity effects too small to be measurable. On the other hand, if one does not impose the above condition a priori, current experiments predict large upper bounds on it, which are compatible with current observations, and may signal the existence of a new length scale. Note that such an intermediate length scale, $\ell_{\text {inter }} \equiv \sqrt{\beta_{0}} \ell_{P l}$ cannot exceed the electroweak length scale $\sim 10^{17} \ell_{P l}$ (as otherwise it would have been observed). This implies $\beta_{0} \leq 10^{34}$. (The factor of 2 in the last term in Eq.(1) follows from Eq.(2) below).

It was shown in [2], that inequality (1) is equivalent to the following modified Heisenberg algebra

$$
\left[x_{i}, p_{j}\right]=i \hbar\left(\delta_{i j}+\beta \delta_{i j} p^{2}+2 \beta p_{i} p_{j}\right) .
$$

This form ensures, via the Jacobi identity, that $\left[x_{i}, x_{j}\right]=$ $0=\left[p_{i}, p_{j}\right][3]$. Next, defining

$$
x_{i}=x_{0 i}, \quad p_{i}=p_{0 i}\left(1+\beta p_{0}^{2}\right)
$$

where $p_{0}^{2}=\sum_{j=1}^{3} p_{0 j} p_{0 j}$ and $x_{0 i}, p_{0 j}$ satisfying the canonical commutation relations $\left[x_{0 i}, p_{0 j}\right]=i \hbar \delta_{i j}$, it is easy to show that Eq.(2) is satisfied to order $\beta$ (henceforth we neglect terms of order $\beta^{2}$ and higher). Here, $p_{0 i}$ can be interpreted as the momentum at low energies (having the standard representation in position space, i.e. $\left.p_{0 i}=-i \hbar d / d x_{i}\right)$, and $p_{i}$ as that at higher energies.

\footnotetext{
*Electronic address: saurya.das@uleth.ca

${ }^{\dagger}$ Electronic address: evagenas@academyofathens.gr
} 
Using (3), any Hamiltonian of the form

$$
H=\frac{p^{2}}{2 m}+V(\vec{r}) \quad\left[\vec{r}=\left(x_{1}, x_{3}, x_{3}\right)\right]
$$

can be written as [4]

$$
\begin{aligned}
H & =H_{0}+H_{1}+\mathcal{O}\left(\beta^{2}\right), \\
\text { where } H_{0} & =\frac{p_{0}^{2}}{2 m}+V(\vec{r}) \text { and } H_{1}=\frac{\beta}{m} p_{0}^{4} .
\end{aligned}
$$

Thus, we see that any system with a well defined quantum (or even classical) Hamiltonian $H_{0}$, is perturbed by $H_{1}$, defined above, near the Planck scale. In other words, Quantum Gravity effects are in some sense universal! It remains to compute the corrections to various phenomena due to the Hamiltonian $H_{1}$. In this article, we study its effects on three such well-understood quantum phenomena, the Lamb shift, the Landau levels and the Scanning Tunnelling Microscope.

\section{The Lamb shift}

For the Hydrogen atom, $V(\vec{r})=-k / r\left(k=e^{2} / 4 \pi \epsilon_{0}=\right.$ $\alpha \hbar c, e=$ electronic charge). To first order, the perturbing Hamiltonian $H_{1}$, shifts the wave-functions to [5]

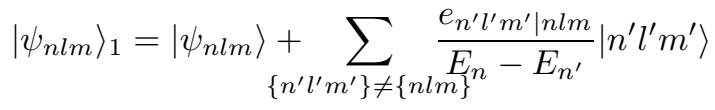

where $n, l, m$ have their usual significance, and $e_{n^{\prime} l^{\prime} m^{\prime} \mid n l m} \equiv\left\langle n^{\prime} l^{\prime} m^{\prime}\left|H_{1}\right| n l m\right\rangle$. Using $p_{0}^{2}=2 m\left[H_{0}+k / r\right]$ [4]

$$
H_{1}=(4 \beta m)\left[H_{0}^{2}+k\left(\frac{1}{r} H_{0}+H_{0} \frac{1}{r}\right)+\left(\frac{k}{r}\right)^{2}\right] .
$$

Thus,

$$
\begin{aligned}
& \frac{e_{n^{\prime} l^{\prime} m^{\prime} \mid n l m}}{4 \beta m}=\left(E_{n}\right)^{2} \delta_{n n^{\prime}} \\
& +k\left(E_{n}+E_{n^{\prime}}\right)\left\langle n^{\prime} l^{\prime} m^{\prime}\left|\frac{1}{r}\right| n l m\right\rangle+k^{2}\left\langle n^{\prime} l^{\prime} m^{\prime}\left|\frac{1}{r^{2}}\right| n l m\right\rangle .
\end{aligned}
$$

It follows from the orthogonality of spherical harmonics that the above are non-vanishing if and only if $l^{\prime}=l$ and $m^{\prime}=m$. Thus, the first order shift in the ground state wave-function is given by (in the position representation)

$$
\begin{aligned}
\Delta \psi_{100}(\vec{r}) & \equiv \psi_{100(1)}(\vec{r})-\psi_{100}(\vec{r})=\frac{e_{200 \mid 100}}{E_{1}-E_{2}} \psi_{200}(\vec{r}) \\
& =\frac{928 \sqrt{2} \beta m E_{0}}{81} \psi_{200}(\vec{r})
\end{aligned}
$$

Next, consider the Lamb shift for the $n^{\text {th }}$ level of the Hydrogen atom [6]

$$
\Delta E_{n}=\frac{4 \alpha^{2}}{3 m^{2}}\left(\ln \frac{1}{\alpha}\right)\left|\psi_{n l m}(0)\right|^{2} .
$$

Varying $\psi_{n l m}(0)$, the additional contribution due to GUP in proportion to its original value is given by

$$
\frac{\Delta E_{n(G U P)}}{\Delta E_{n}}=2 \frac{\Delta\left|\psi_{n l m}(0)\right|}{\psi_{n l m}(0)} .
$$

Thus, for the Ground State, using $\psi_{100}(0)=a_{0}^{-3 / 2} \pi^{-1 / 2}$ and $\psi_{200}(0)=a_{0}^{-3 / 2}(8 \pi)^{-1 / 2}$, where $a_{0}$ is the Bohr radius, we get

$$
\begin{aligned}
\frac{\Delta E_{0(G U P)}}{\Delta E_{0}} & =2 \frac{\Delta\left|\psi_{100}(0)\right|}{\psi_{100}(0)}=\frac{928 \beta m E_{0}}{81} \\
& \approx 10 \beta_{0} \frac{m}{M_{P l}} \frac{E_{0}}{M_{P l} c^{2}} \approx 0.47 \times 10^{-48} \beta_{0} .
\end{aligned}
$$

The above result may be interpreted in two ways. First, if one assumes $\beta_{0} \sim 1$, then it predicts a non-zero, but virtually unmeasurable effect of Quantum Gravity/GUP. On the other hand, if such an assumption is not made, the current accuracy of precision measurement of Lamb shift of about 1 part in $10^{12}[4,7]$, sets the following upper bound on $\beta_{0}$

$$
\beta_{0}<10^{36} .
$$

This bound is weaker than that set by the electroweak scale, but not incompatible with it. Moreover, with more accurate measurements in the future, this bound is expected to get reduced by several orders of magnitude, in which case, it could signal a new and intermediate length scale between the electroweak and the Planck scale.

\section{The Landau Levels}

Next consider a particle of mass $m$ and charge $e$ in a constant magnetic field $\vec{B}=B \hat{z}$, described by the vector potential $\vec{A}=B x \hat{y}$ and the Hamiltonian

$$
\begin{aligned}
H_{0} & =\frac{1}{2 m}\left(\vec{p}_{0}-e \vec{A}\right)^{2} \\
& =\frac{p_{0 x}^{2}}{2 m}+\frac{p_{0 y}^{2}}{2 m}-\frac{e B}{m} x p_{0 y}+\frac{e^{2} B^{2}}{2 m} x^{2} .
\end{aligned}
$$

Since $p_{0 y}$ commutes with $H$, replacing it with its eigenvalue $\hbar k$, we get

$$
H_{0}=\frac{p_{0 x}^{2}}{2 m}+\frac{1}{2} m \omega_{c}^{2}\left(x-\frac{\hbar k}{m \omega_{c}}\right)^{2},
$$

where $\omega_{c}=e B / m$ is the the cyclotron frequency. This is nothing but the Hamiltonian of a harmonic oscillator in $x$ direction, with its equilibrium position given by $x_{0} \equiv \hbar k / m \omega_{c}$. Consequently, the eigenfunctions and eigenvalues are given by

$$
\begin{aligned}
\psi_{k, n}(x, y) & =e^{i k y} \phi_{n}\left(x-x_{0}\right) \\
E_{n} & =\hbar \omega_{c}\left(n+\frac{1}{2}\right), n \in N,
\end{aligned}
$$

where $\phi_{n}$ are the harmonic oscillator wave-functions.

Following the procedure outlined in the introduction, the GUP-corrected Hamiltonian assumes the form

$$
\begin{aligned}
H & =\frac{1}{2 m}\left(\vec{p}_{0}-e \vec{A}\right)^{2}+\frac{\beta}{m}\left(\vec{p}_{0}-e \vec{A}\right)^{4} \\
& =H_{0}+4 \beta m H_{0}^{2}
\end{aligned}
$$


where in the last step we have used Eq.(14). Evidently, the eigenfunctions remain unchanged, which alone guarantees for example, that the GUP will have no effect at all on phenomena such as the Quantum Hall Effect, the Bohm-Aharonov effect, and Dirac Quantization. However, the eigenvalues shift by

$$
\begin{aligned}
& \Delta E_{n(G U P)}=4 \beta m\left\langle\phi_{n}\left|H_{0}^{2}\right| \phi_{n}\right\rangle=4 \beta m\left(\hbar \omega_{c}\right)^{2}\left(n+\frac{1}{2}\right)^{2}, \\
& \text { or } \frac{\Delta E_{n(G U P)}}{E_{n}}=4 \beta m\left(\hbar \omega_{c}\right)\left(n+\frac{1}{2}\right) \approx \beta_{0} \frac{m}{M_{P l}} \frac{\hbar \omega_{c}}{M_{P l} c^{2}} .
\end{aligned}
$$

For an electron in a magnetic field of $10 \mathrm{~T}, \omega_{\mathrm{c}} \approx 10^{3} \mathrm{GHz}$ and we get

$$
\frac{\Delta E_{n(G U P)}}{E_{n}} \approx 2.30 \times 10^{-54} \beta_{0} .
$$

Once again, if $\beta_{0} \sim 1$, this correction is too small to be measured. Without this assumption, an accuracy of 1 part in $10^{3}$ in direct measurements of Landau levels using a STM (which is somewhat over-optimistic) [8], the upper bound on $\beta_{0}$ follows

$$
\beta_{0}<10^{50} .
$$

This bound is far weaker than that set by electroweak measurements, but compatible with the latter (as was the case for the Lamb shift). Once again, better accuracy should tighten this bound, and perhaps predict an intermediate length scale.

\section{Potential Barrier and STM}

In a STM, electrons of energy $E$ (close to the Fermi energy) from a metal tip at $x=0$, tunnel quantum mechanically to a sample surface a small distance away at $x=a$. This gap, (across which a bias voltage may be applied), is associated with a potential barrier of height $V_{0}>E[9]$. Thus

$$
V(x)=V_{0}[\theta(x)-\theta(x-a)],
$$

where $\theta(x)$ is the usual step function. The wave-functions for the three regions, namely $x \leq 0,0 \leq x \leq a$, and $x \geq a$, are $\psi_{1}, \psi_{2}$, and $\psi_{3}$, respectively, and satisfy the following GUP corrected Schrödinger equation $\left(d^{n} \equiv d^{n} / d x^{n}\right)$

$$
\begin{aligned}
& d^{2} \psi_{1,3}+k^{2} \psi_{1,3}-\ell_{P l}^{2} d^{4} \psi_{1,3}=0, \\
& d^{2} \psi_{2}-k_{1}^{2} \psi_{2}-\ell_{P l}^{2} d^{4} \psi_{2}=0
\end{aligned}
$$

where $k=\sqrt{2 m E / \hbar^{2}}, k_{1}=\sqrt{2 m\left(V_{0}-E\right) / \hbar^{2}}$. The solutions to the above to leading order in $\ell_{P l}, \beta$ are

$$
\begin{aligned}
& \psi_{1}=A e^{i k^{\prime} x}+B e^{-i k^{\prime} x}+A_{1} e^{x / \ell_{P l}} \\
& \psi_{2}=F e^{k_{1}^{\prime} x}+G e^{-k_{1}^{\prime} x}+H_{1} e^{x / \ell_{P l}}+L_{1} e^{-x / \ell_{P l}} \\
& \psi_{3}=C e^{i k^{\prime} x}+D_{1} e^{-x / \ell_{P l}}
\end{aligned}
$$

where $k^{\prime} \equiv k\left(1-\beta \hbar^{2} k^{2}\right)$ and $k_{1}^{\prime} \equiv k_{1}\left(1-\beta \hbar^{2} k_{1}^{2}\right)$. Note the appearance of new exponential terms, which drop out in the $\ell_{P l} \rightarrow 0$ limit. In the above, we have omitted the left-mover from $\psi_{3}$ and the exponentially growing terms from both $\psi_{1}$ and $\psi_{3}$. The boundary conditions

$$
\begin{aligned}
& \left.d^{n} \psi_{1}\right|_{x=0}=\left.d^{n} \psi_{2}\right|_{x=0} \quad, n=0,1,2,3 \\
& \left.d^{n} \psi_{2}\right|_{x=a}=\left.d^{n} \psi_{3}\right|_{x=a} \quad, n=0,1,2,3
\end{aligned}
$$

on wave-functions (24-26) yield the following transmission coefficient

$$
T=\left|\frac{C}{A}\right|^{2}=\left[1+\frac{\left(k^{\prime 2}+k_{1}^{\prime 2}\right)^{2} \sinh ^{2}\left(k_{1}^{\prime} a\right)}{\left(2 k^{\prime} k_{1}^{\prime}\right)^{2}}\right]^{-1} .
$$

The reflection coefficient $R=|B / A|^{2}=1-T$. Using Eq.(29) and the definitions of $k, k_{1}, k^{\prime}, k_{1}^{\prime}$, it can be shown that when $k_{1} a \gg 1$, which is the limit relevant for STMs, the transmission coefficient is approximately

$$
\begin{gathered}
T=T_{0}\left[1+\frac{4 m \beta\left(2 E-V_{0}\right)^{2}}{V_{0}}+\frac{2 \beta a}{\hbar}\left[2 m\left(V_{0}-E\right)\right]^{\frac{3}{2}}\right] \\
\text { where } T_{0}=\frac{16 E\left(V_{0}-E\right)}{V_{0}^{2}} e^{-2 k_{1} a}
\end{gathered}
$$

$T_{0}$ being the standard tunnelling amplitude. The current $I$ flowing from the tip to the sample is proportional to $T$, and is usually magnified using an amplifier of gain $\mathcal{G}$. From Eq.(30) the enhancement in current due to GUP is given by

$$
\begin{aligned}
& \frac{\delta I}{I_{0}}=\frac{\delta T}{T_{0}}=4 \beta \frac{m\left(2 E-V_{0}\right)^{2}}{V_{0}}+\frac{2 \beta a}{\hbar}\left[2 m\left(V_{0}-E\right)\right]^{\frac{3}{2}} \\
= & \frac{4 \beta_{0} m}{M_{P l}} \frac{\left(2 E-V_{0}\right)^{2}}{V_{0} M_{P l} c^{2}}+\frac{4 \sqrt{2} \beta_{0} a}{\ell_{P l}}\left(\frac{m}{M_{P l}}\right)^{\frac{3}{2}}\left(\frac{V_{0}-E}{M_{P l} c^{2}}\right)^{\frac{3}{2}} .
\end{aligned}
$$

Then, assuming the following approximate (but realistic) values [9]

$$
\begin{aligned}
& m=m_{e}=0.5 \mathrm{MeV} / \mathrm{c}^{2}, E \approx V_{0}=10 \mathrm{eV}, \\
& a=10^{-10} \mathrm{~m}, I_{0}=10^{-9} \mathrm{~A}, \mathcal{G}=10^{9},
\end{aligned}
$$

we get

$$
\frac{\delta I}{I_{0}}=\frac{\delta T}{T_{0}}=10^{-48} \beta_{0} \text { and } \delta \mathcal{I} \equiv \mathcal{G} \delta I=10^{-48} \beta_{0} A .
$$

Thus, for the GUP induced excess current $\delta \mathcal{I}$ to add up to the charge of just one electron, $e \approx 10^{-19} C$, one would have to wait for a time

$$
\tau=\frac{e}{\delta \mathcal{I}}=10^{29} \beta_{0}^{-1} s .
$$

If $\beta_{0} \sim 1$, this is far greater than the age of our universe $\left(10^{18} \mathrm{~s}\right)$. However, if the quantity $\delta \mathcal{I}$ can be increased by a factor of about $10^{21}$, say by a combination of increase in $I$ and $\mathcal{G}$, and by a larger value of $\beta_{0}$, the above time will be reduced to about a year $\left(\approx 10^{8} s\right)$, and one can hope to measure the effect of GUP. Conversely, if such a GUP induced current cannot be measured in such a time-scale, it will put an upper bound

$$
\beta_{0}<10^{21} \text {. }
$$


Note that this is more stringent than the two previous examples, and is in fact consistent with that set by the electroweak scale! In practice however, it may be easier to experimentally determine the apparent barrier height $\Phi_{A} \equiv V_{0}-E$, and the (logarithmic) rate of increase of current with the gap. From Eq.(30) they are related by $[9]$

$$
\sqrt{\Phi_{A}}=\frac{\hbar}{\sqrt{8 m}}\left|\frac{d \ln I}{d a}\right|\left(1-\frac{\beta \hbar^{2}}{4}\left|\frac{d \ln I}{d a}\right|^{2}\right)
$$

The cubic deviation from the linear $\sqrt{\Phi_{A}}$ vs $\left|\frac{d \ln I}{d a}\right|$ curve predicted by GUP may be easier to spot and the value of $\beta$ estimated with improved accuracies.

To summarize, our results indicate that either the predictions of GUP are too small to measure at present $\left(\beta_{0} \sim 1\right)$, or that they signal a new intermediate length scale $\left(\beta_{0} \gg 1\right)$. It is not inconceivable that such a new length scale may show up in future experiments in the
Large Hadron Collider. Perhaps more importantly, our study reveals the universality of GUP effects, meaning that the latter can potentially be tested in a wide class of quantum mechanical systems, of which we have studied just a handful here. Promising areas include statistical systems (where a large number of particles may offset the smallness of $\beta$ ), study of whether normally forbidden transitions and processes can be allowed by the GUP corrected Hamiltonian, and processes which may get corrected by a fractional power of $\beta_{0}$. We hope to report on some of these in the near future. In the best case scenario, this could open a much needed low-energy 'window' to Quantum Gravity Phenomenology.

\section{Acknowledgment}

We thank K. Ali, B. Belchev, A. Dasgupta, R. B. Mann, S. Sur and M. Walton for useful discussions. This work was supported in part by the Natural Sciences and Engineering Research Council of Canada and by the Perimeter Institute for Theoretical Physics.
[1] D. Amati, M. Ciafaloni, G. Veneziano, Phys. Lett. B 216 (1989) 41; M. Maggiore, Phys. Lett. B 304 (1993) 65 [arXiv:hep-th/9301067]; L. J. Garay, Int. J. Mod. Phys. A 10 (1995) 145 [arXiv:gr-qc/9403008].

[2] A. Kempf, G. Mangano, R. B. Mann, Phys. Rev. D52 (1995) 1108 [arXiv:hep-th/9412167].

[3] A. Kempf, J.Phys. A 30 (1997) 2093 [arXiv:hepth/9604045].

[4] F. Brau, J. Phys. A 32 (1999) 7691 [arXiv:quantph/9905033].

[5] B. H. Bransden, C. J. Joachain, Quantum Mechanics
(Benjamin Cummings, Harlow, U.K., 2000), 2nd ed.

[6] J. D. Bjorken, S. D. Drell, Relativistic Quantum Mechanics (Mc-Graw Hill, New York, 1964), p.60.

[7] G. Newton, D. A. Andrews, P. J. Unsworth, Phil. Trans. Roy. Soc. Lond. Series A, Math. and Phys. Sc., 290, No. 1373 (1979), 373.

[8] J. W. G. Wildöer, C. J. P. M. Harmans, H. van Kempen, Phys. Rev. B 55 (1997) R16013.

[9] J. A. Stroscio, W. J. Kaiser, Scanning Tunneling Microscopy (Academic Press, New York, 1993). 